\title{
Downregulation of IncRNA PMS2L2 in patients with gastric adenocarcinoma predicts poor prognosis
}

\author{
JUNPING BIAN, GUANGCHUN LI, ZHEN ZHANG and BIN LIU \\ Department of Gastroenterology, The Second Hospital of Shandong University, Jinan, Shandong 250033, P.R. China
}

Received February 19, 2019; Accepted January 8, 2020

DOI: 10.3892/ol.2020.11578

\begin{abstract}
Long non-coding RNA PMS1 homolog 2 mismatch repair system component pseudogene 2 (PMS2L2) is a key player in lipopolysaccharide-induced inflammatory responses. Preliminary deep sequencing data revealed that PMS2L2 was downregulated in gastric adenocarcinoma (GA) tissues compared with healthy adjacent tissues and the aim of the present study was to investigate the role of PMS2L 2 in GA. In the present study, reverse transcription-quantitative PCR assays were performed to analyze gene expression. Cell transfections were performed to analyze gene interactions and Transwell assays were performed to analyze cell invasion and migration. The results revealed that PMS2L2 expression was downregulated in cancer tissues obtained from patients with GA compared with healthy adjacent tissues and was not significantly affected by clinical stage. Furthermore, low levels of PMS2L2 in cancer tissues were closely associated with a low overall 5-year survival rate in patients. MicroRNA (miR)-25 was upregulated in GA tissues compared with healthy adjacent tissues and inversely associated with PMS2L2 levels. In GA cells in vitro, overexpression of PMS2L2 downregulated the expression of miR-25, while miR-25 overexpression did not significantly affect PMS2L2 expression. Furthermore, PMS2L2 overexpression inhibited the migration and invasion of GA cells. miR-25 overexpression partially rescued the decreased migration and invasion of GA cells caused by PMS2L2 overexpression. Therefore, PMS2L2 may downregulate miR-25 expression to inhibit GA.
\end{abstract}

\section{Introduction}

Gastric cancer is a common malignancy worldwide that affects $>1 \%$ of the worldwide population (1). A large number

Correspondence to: Dr Bin Liu, Department of Gastroenterology, The Second Hospital of Shandong University, 247 Bei Yuan Street, Jinan, Shandong 250033, P.R. China

E-mail:vx07412@163.com

Key words: gastric adenocarcinoma, long non-coding RNA PMS1 homolog 2 mismatch repair system component pseudogene 2, microRNA-25, prognosis of patients with gastric cancer are diagnosed with inoperable tumors or experience high recurrence rates following curative resection (2). An unhealthy diet, cigarette smoking and alcohol consumption have been identified as risk factors for gastric cancer and reduced exposure to risk factors significantly reduces the incidence of the disease (3-5). It has been revealed genetic alterations as risk factors for gastric cancer (3-6); however, the limited number of oncogenes and tumor suppressors currently identified do not sufficiently explain the complex pathogenesis of gastric cancer (6).

Previous studies have shown that long non-coding RNAs (lncRNAs), transcripts $>200$ nucleotides in length, are not transcriptional 'noise', but serve important roles in growth and developmental processes $(7,8)$. IncRNAs regulate gene expression at multiple levels, and altered expression of lncRNAs may result in dysregulated gene expression, thereby contributing to the occurrence of diseases, such as cancer $(9,10)$. Therefore, characterization of the functions of lncRNAs may provide insights into disease prevention and treatment. However, the function of the majority of predicted or isolated lncRNAs remains unclear. PMS1 homolog 2 mismatch repair system component pseudogene 2 (PMS2L2) is a recently identified IncRNA that exhibits protective effects in chondrocytes during lipopolysaccharide-induced inflammation (11). Preliminary deep sequencing data revealed that PMS2L2 expression was downregulated in gastric adenocarcinoma (GA) tissues in comparison with adjacent non-tumor tissues and was inversely associated with microRNA (miR/miRNA)-25 expression levels (data not shown). A previous study revealed that miR-25 expression was upregulated in GA tissues compared with adjacent non-tumor tissues and promoted the in vitro proliferation, invasion and migration of GA cells (12). Furthermore, high levels of miR-25 predicted poor prognosis of patients with GA. Therefore, the present study investigated the role of PMS2L2 in GA and its potential interactions with miR-25.

\section{Materials and methods}

Specimens and patients. Tissue specimens, including GA and healthy adjacent non-cancerous tissues (within $2 \mathrm{~cm}$ of the tumor margin), were obtained from 72 patients with GA at The Second Hospital of Shandong University between May 2010 and May 2015. Histopathological examination revealed that the cancer cell content in all adjacent non-cancerous tissues was 
$<1 \%$. The patients included 38 males and 34 females (age range, 33-68 years; mean age, 52.2 \pm 8.3 years). The inclusion criteria were as follows: i) New GA cases diagnosed by histopathological examination; ii) no previous history of malignancies; and iii) no familial history of malignancies. The exclusion criteria were as follows: i) Patients transferred from other hospitals; ii) patients who received treatment within 3 months prior to admission; and iii) patients with co-morbidities. Based on the criteria established by the American Joint Committee on Cancer (13), there were 14, 16, 28 and 14 patients with stage I-IV cancer, respectively. The present study was approved by the Ethics Committee of The Second Hospital of Shandong University and all patients provided written informed consent.

Follow-up. A 5-year follow-up study was performed to monitor the survival rate of the patients. Patients visited the outpatients department or were monitored remotely over the telephone every month. Patients who succumbed to other clinical disorders, including heart disease, or accidents, including traffic accidents, were excluded from the study. The aforementioned 72 patients completed the follow-up study.

Cell lines and cell culture. The human GA cell line AGS (Sigma-Adrich; Merck KGaA) was used to perform all in vitro experiments. The normal gastric cell line Hs 738.St/Int (cat. no. CRL-7869 ${ }^{\mathrm{TM}}$; American Type Culture Collection) served as healthy control cells. Cells were cultured in F-12K medium (Sigma-Adrich; Merck KGaA) supplemented with $10 \%$ fetal bovine serum (FBS; Sigma-Adrich; Merck KGaA) and maintained at $37^{\circ} \mathrm{C}$ and $5 \% \mathrm{CO}_{2}$.

Transient transfections. The PMS2L2 expression vector was constructed by inserting full length PMS2L2 cDNA into a pcDNA3.1 vector (Sangon Biotech Co., Ltd.). Negative control (NC) miRNA (5'-CUAGUCGUGUAU ACAGUGUGA-3') and the miR-25 mimic (5'-CAUUGC ACUUGUCUCGGUCUGA-3') were purchased from Sigma-Aldrich, Merck KGaA. All cell transfections were performed using Lipofectamine ${ }^{\circledR} 2000$ (Thermo Fisher Scientific, Inc.) and $10 \mathrm{nM}$ vector and $35 \mathrm{nM}$ miRNA with all procedures performed following the manufacturer's instructions. Subsequent experiments were performed $24 \mathrm{~h}$ post-transfection. Negative controls included cells transfected with an empty vector or NC miRNA and controls included untransfected cells.

Reverse-transcription quantitiative polymerse chain reaction (RT-qPCR). Total RNA was extracted from GA and healthy adjacent non-cancerous tissues as well as the AGS and Hs 738.St/Int cell lines using RNAzol reagent (Sigma-Aldrich; Merck KGaA). Total RNA was reverse transcribed into cDNA using AMV Reverse Transcriptase XL (Clontech Laboratories, Inc.). The expression of PMS2L2 was analyzed by qPCR using the DyNAmo Flash SYBR Green qPCR kit (Thermo Fisher Scientific, Inc.) and 18S rRNA as an endogenous control.

The miRNA isolation kit (cat. no. RMI050; Geneaid Biotech Ltd.) was used to extract miRNA from AGS and Hs 738.St/Int cell lines. The TaqMan MicroRNA Reverse Transcription kit (Thermo Fisher Scientific, Inc.) was used to perform reverse transcription. The expression of miR-25 was

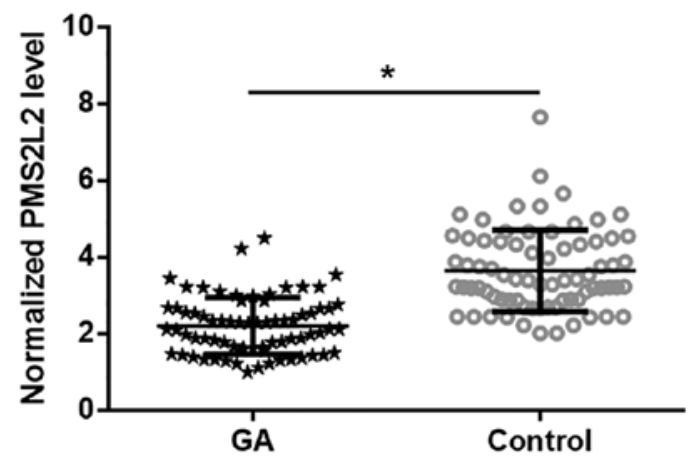

Figure 1. PMS2L2 was downregulated in GA. PMS2L2 expression data were analyzed by the paired t-test. PMS2L 2 was significantly downregulated in GA tissues compared with healthy adjacent tissues. ${ }^{*} \mathrm{P}<0.05$, as indicated. PMS2L2, PMS1 homolog 2 mismatch repair system component pseudogene 2; GA, gastric adenocarcinoma.

analyzed by qPCR using the TaqMan Real-Time PCR Master mix (Thermo Fisher Scientific, Inc.) and U6 as an endogenous control.

Primer sequences were: PMS2L2 forward, 5'-AGTCTA AGCACTGCGGTGAA-3' and reverse, 5'-GGTAGAAAT GGTGACATCAT-3'; 18S rRNA forward, 5'-CTACCACAT CCAAGGAAGCA-3' and reverse, 5'-TTTTTCGTCACT ACCTCCCCG-3'. The forward primer sequence of miR-25 was: 5'-CATTGCACTTGTCTCGGTC-3'. Universal reverse primers and U6 forward primer were from the kit. The thermocycling conditions were: $95^{\circ} \mathrm{C}$ for $1 \mathrm{~min}$, then 40 cycles of $95^{\circ} \mathrm{C}$ for $10 \mathrm{sec}$ and $60^{\circ} \mathrm{C}$ for $50 \mathrm{sec}$. All qPCR reactions were performed in triplicate and the $2^{-\Delta \Delta \mathrm{Cq}}$ method (14) was used to quanitfy expression levels.

Cell migration and invasion. AGS and Hs 738.St/Int cells were harvested $24 \mathrm{~h}$ post-transfection. A single cell suspension was prepared using serum-free F-12K medium and the final cell density was adjusted to $3 \times 10^{4}$ cells $/ \mathrm{ml}$. A total of $0.1 \mathrm{ml}$ cell suspension was plated in the upper chamber of Corning Transwell Cell Culture Plate Insert $(8.0 \mu \mathrm{m}$ pore; Corning, Inc.). The lower chamber was filled with F-12K medium (supplemented with 20\% FBS). For invasion assays, the Transwell membranes were coated with Matrigel (EMD Millipore) for $6 \mathrm{~h}$ at $37^{\circ} \mathrm{C}$. Following incubation at $37^{\circ} \mathrm{C}$ for $2.5 \mathrm{~h}$, cells were fixed in ice-cold $70 \%$ ethanol for $20 \mathrm{~min}$ at $4{ }^{\circ} \mathrm{C}$. The migratory cells were then stained with $0.5 \%$ crystal violet (Sigma-Aldrich; Merck KGaA) for 16 min at room temperature. Stained cells were observed under an optical microscope and cells of five randomly selected visual fields were counted (magnification, $x 40$ ). The control groups (untransfected cells) were set to $100 \%$ and all other groups were normalized to the control groups.

Statistical analysis. Data are presented as the mean \pm standard deviation values calculated from experiments performed in triplicate. GraphPad prism 6 (GraphPad Software, Inc.) was used to perform all statistical analysis. Differences between GA tissues and healthy adjacent tissues were analyzed by the paired t-test. Differences among cell transfection groups or clinical stages were analyzed by the one-way ANOVA followed by Tukey's post hoc test. Associations between PMS2L2 and 

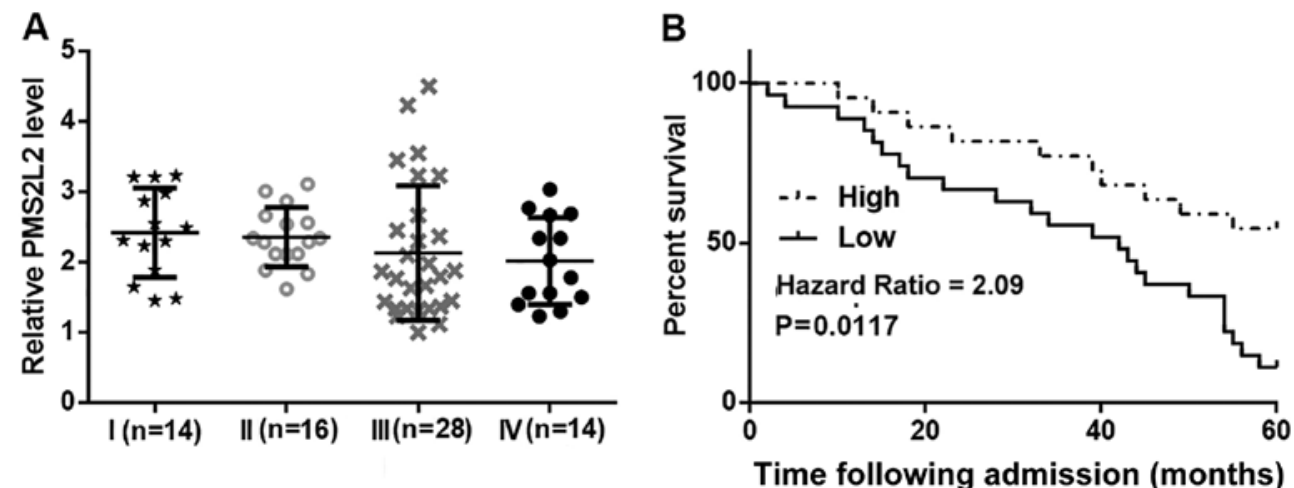

Figure 2. PMS2L2 was not affected by clinical stage but predicted the survival rate. (A) Analysis of PMS2L2 expression data by the one-way ANOVA followed by Tukey's post hoc test revealed no significant differences in PMS2L2 levels in patients with different clinical stages. (B) Survival curve analysis showed that low levels of PMS2L2 in gastric adenocarcinoma tissues were closely associated with a low overall 5-year survival rate. PMS2L2, PMS1 homolog 2 mismatch repair system component pseudogene 2 .
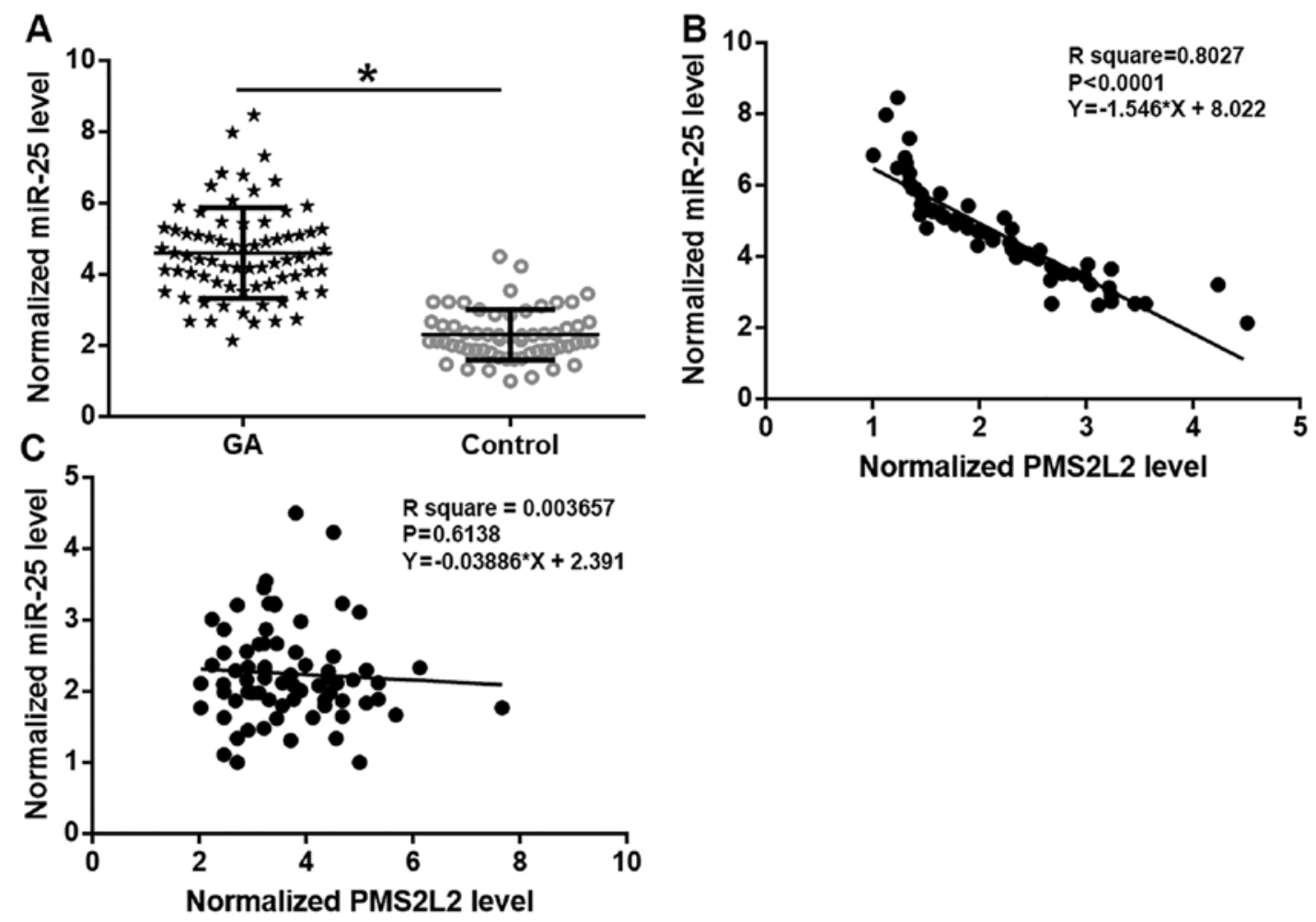

Figure 3. miR-25 was upregulated in GA and inversely associated with PMS2L2. miR-25 expression data were analyzed by the paired t-test. (A) miR-25 was significantly upregulated in GA tissues compared with healthy adjacent tissues. Associations between PMS2L2 and miR-25 were analyzed by linear regression and the results showed that miR-25 was inversely associated with PMS2L2 in (B) GA tissues but not in (C) healthy adjacent tissues. "P<0.05, as indicated. miR, microRNA; GA, gastric adenocarcinoma; PMS2L2, PMS1 homolog 2 mismatch repair system component pseudogene 2.

miR-25 were analyzed by linear regression. Patients were grouped into high $(\mathrm{n}=33)$ and low $(\mathrm{n}=39)$ PMS2L2 level groups based on Youden's index (cut-off value=2.14). Survival curves were plotted using the Kaplan-Meier method and compared by the log-rank test. $\mathrm{P}<0.05$ was used to indicate a statistically significant difference.

\section{Results}

$P M S 2 L 2$ is downregulated in GA. PMS2L2 expression in tissues was detected by RT-qPCR. The GA tissue with the lowest expression level was set to ' 1 ', and all other samples were normalized to this sample. Expression data were analyzed by the paired t-test. PMS2L2 was significantly downregulated in GA tissues compared with healthy adjacent tissues (Fig. 1; $\mathrm{P}<0.05)$.

PMS2L2 is not affected by clinical stage but is associated with survival rate. PMS2L2 expression in GA tissues obtained from patients with different clinical stages was compared by the one-way ANOVA and Tukey's post hoc test. There were no significant differences in PMS2L2 levels among these groups of patients (Fig. 2A). Subsequently, patients were grouped into high $(n=33)$ and low $(n=39)$ PMS2L2 expression level groups 

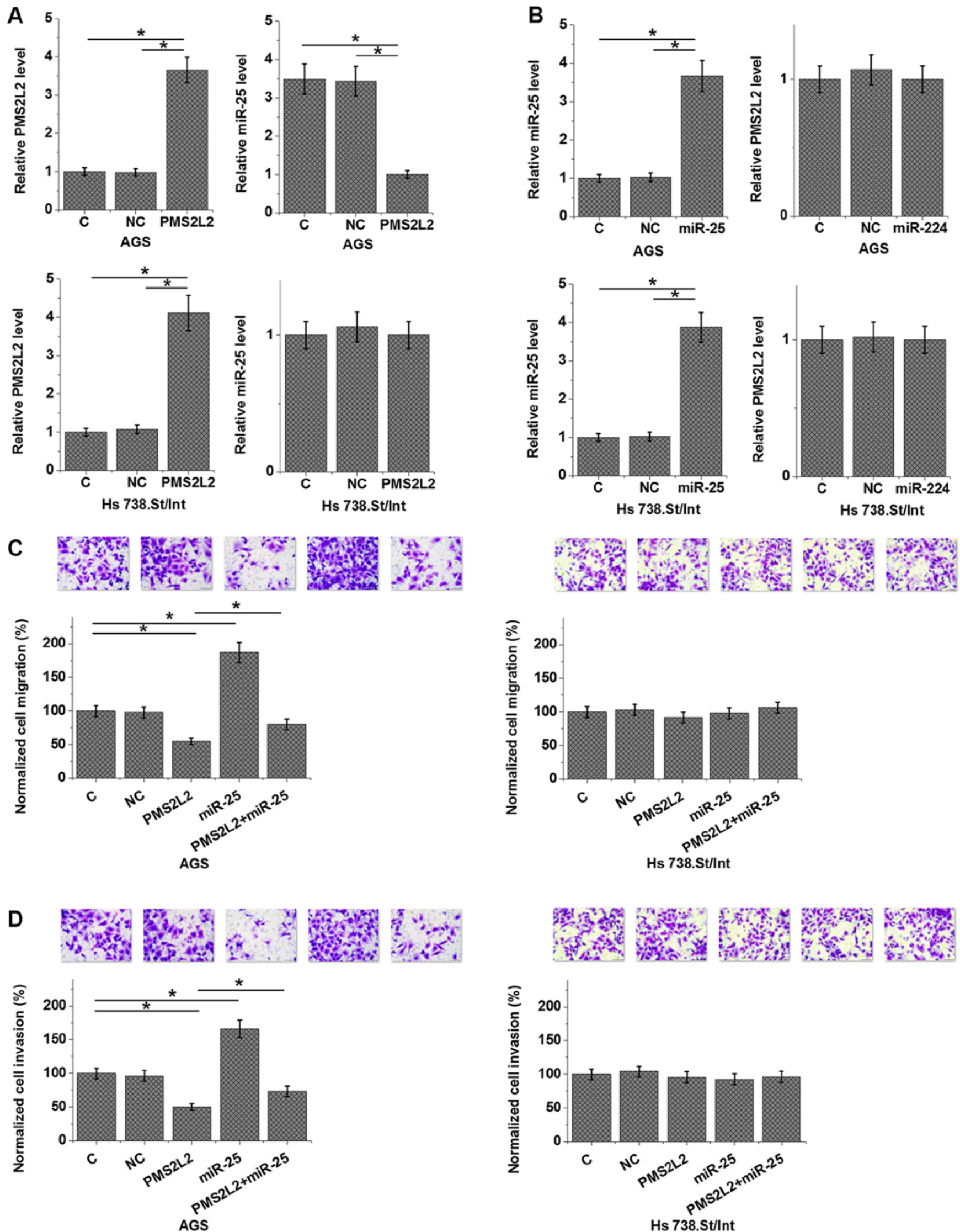

Figure 4. PMS2L2 downregulated miR-25 to regulate the migration and invasion of gastric adenocarcinoma cells, but not normal gastric tissue cells. Compared with the C and NC cells, cells transfected with a PMS2L2 overexpression vector and an miR-25 mimic exhibited significantly upregulated (A) PMS2L2 and (B) miR-25 levels $24 \mathrm{~h}$ post-transfection. Furthermore, overexpression of PMS2L2 downregulated the expression of miR-25 in AGS cells but not in Hs 738 . St/Int, while miR-25 overexpression did not significantly affect PMS2L2 expression in both cell lines. Transwell assays revealed that PMS2L2 overexpression inhibited the (C) migration and (D) invasion of AGS cells but not Hs 738.St/Int cells. Moreover, miR-25 overexpression partially rescued the decreased migration and invasion of AGS cells caused by PMS2L2 overexpression. "P $<0.05$, as indicated. PMS2L2, PMS1 homolog 2 mismatch repair system component pseudogene 2; miR, microRNA; C, control; NC, negative control. 
based on Youden's index. As shown in Fig. 2B, patients with low levels of PMS2L2 exhibited a significantly shorter survival rate compared with patients with high expression $(\mathrm{P}=0.0117)$.

miR-25 is upregulated in GA and inversely associated with PMS2L2. miR-25 expression in tissues was detected by RT-qPCR. The GA tissue with the lowest expression level was set to ' 1 ', and all other samples were normalized to this sample. Expression data were analyzed by the paired t-test. miR-25 was significantly upregulated in GA tissues compared with healthy adjacent tissues (Fig. 3A; P<0.05). Associations between PMS2L2 and miR-25 were analyzed by linear regression. PMS2L2 and miR-25 expression levels were significantly inversely associated in GA tissues (Fig. 3B, $\mathrm{R}^{2}=0.8207$, $\mathrm{P}<0.0001$ ) but not in healthy adjacent tissues (Fig. 3C $\left.\mathrm{R}^{2}=0.003657, \mathrm{P}=0.6138\right)$.

PMS2L2 downregulates miR-25 to regulate the migration and invasion of GA cells but not normal gastric tissue cells. A PMS2L2 overexpression vector and miR-25 mimic were transfected into AGS and Hs 738.St/Int cells, and compared with the NC and control cells, PMS2L2 (Fig. 4A) and miR-25 (Fig. 4B) expression levels were significantly upregulated $24 \mathrm{~h}$ post-transfection (both $\mathrm{P}<0.05$ ). In addition, overexpression of PMS2L2 downregulated the expression of miR-25 in AGS cells but not in Hs 738.St/Int (Fig. 4A; P<0.05), while miR-25 overexpression did not significantly affect PMS2L2 expression in both cell lines (Fig. 4B, both $\mathrm{P}>0.05$ ). Transwell migration and invasion assays revealed that PMS2L2 overexpression inhibited migration (Fig. 4C) and invasion (Fig. 4D) of AGS cells (both $\mathrm{P}<0.05$ ) but not Hs 738.St/Int cells. Moreover, miR-25 overexpression partially rescued the decreased migration and invasion of AGS cells caused by PMS2L2 overexpression.

\section{Discussion}

The present study investigated the role of PMS2L2 in GA. PMS2L2 was downregulated in GA tissues compared with healthy adjacent tissues and had prognostic value. Moreover, the results suggested that PMS2L2 may inhibit the migration and invasion of GA cells by downregulating miR-25, which is an oncogenic miRNA in this disease (12).

The prognosis and outlook of patients with GA is worse in China compared with developed countries (15). It has been revealed that lncRNAs serve important roles in GA (16). For example, IncRNA ncRuPAR inhibited protease-activated receptor-1 to suppress the development of GA (16). Therefore, the altered expression of IncRNAs may be associated with the survival time of patients with GA. The present study revealed that PMS2L2 expression was downregulated in GA tissues compared with healthy adjacent tissues. Interestingly, the level PMS2L2 expression in GA tissues was not significantly affected by the clinical stage of GA. Therefore, PMS2L2 is likely to be involved in the overall process of GA rather than specific stages. Furthermore, low expression of PMS2L2 in GA was closely associated with poor survival rate. Therefore, PMS2L2 expression may be used to predict the survival of patients with GA and aid the development of individualized treatment.

Preliminary deep sequencing data revealed that PMS2L2 and miR-25 were inversely associated in GA tissues (data not shown). miR-25 is a well-characterized oncogenic microRNA and participates in several aspects of cancer development, including the regulation of cancer cell behavior and the responses of cancer cells to chemotherapy (17-19). The results of the present study suggested that PMS2L2 is likely to be an upstream inhibitor of miR-25 in GA cells, and the modulation of miR-25 by PMS2L 2 is involved in the regulation of migration and invasion of GA cells but not normal gastric tissues cells. Therefore, PMS2L2 may serve as a potential therapeutic target for GA. It is known that lncRNAs can inhibit the function of miRNAs by serving as sponges $(20,21)$. In effect, PMS2L2 sponges miR-203 and serves a protective role in chondrocytes protect against apoptosis (11). However, PMS2L2 does not sponge miR-25 as an appropriate binding site has not been identified (data not shown). In the present study, PMS2L2 and miR-25 were not significantly associated in healthy adjacent tissues. In addition, PMS2L2 overexpression did not affect miR-25 expression in normal gastric cancer cells. Therefore, the interaction between PMS2L2 and miR-25 is likely indirect or GA-specific. Future studies are required to identify the disease-related factors that mediate the interaction between PMS2L2 and miR-25.

In conclusion, the present study revealed that PMS2L2 was downregulated in GA and PMS2L2 may downregulate miR-25 to inhibit the migration and invasion of GA cells.

\section{Acknowledgements}

Not applicable.

\section{Funding}

No funding was received.

\section{Availability of data and materials}

The datasets used and/or analyzed during the current study are available from the corresponding author on reasonable request.

\section{Authors' contributions}

JB and BL designed the experiments. JB and GL performed experiments. ZZ collected and analyzed data. BL drafted the manuscript. All authors read and approved the final manuscript.

\section{Ethics approval and consent to participate}

The present study was approved by the Ethics Committee of The Second Hospital of Shandong University and all patients provided written informed consent.

\section{Patient consent for publication}

Not applicable.

\section{Competing interests}

The authors declare that they have no competing interests. 


\section{References}

1. Hartgrink HH, Jansen EPM, van Grieken NC and van de Velde CJ: Gastric cancer. Lancet 374: 477-490, 2009.

2. Van Cutsem E, Sagaert X, Topal B, Haustermans K and Prenen H: Gastric cancer. Lancet 388: 2654-2664, 2016.

3. Fang X, Wei J, He X, An P, Wang H, Jiang L, Shao D, Liang H, Li Y, Wang F and Min J: Landscape of dietary factors associated with risk of gastric cancer: A systematic review and dose-response meta-analysis of prospective cohort studies. Eur J Cancer 51: 2820-2832, 2015.

4. Praud D, Rota M, Pelucchi C, Bertuccio P, Rosso T, Galeone C, Zhang ZF, Matsuo K, Ito $\mathrm{H}, \mathrm{Hu} \mathrm{J}$, et al: Cigarette smoking and gastric cancer in the Stomach Cancer Pooling (StoP) Project. Eur J Cancer Prev 27: 124-133, 2018.

5. Ma K, Baloch Z, He TT and Xia X: Alcohol consumption and gastric cancer risk: A meta-analysis. Med Sci Monit 23: 238-246, 2017.

6. Mocellin S, Verdi D, Pooley KA and Nitti D: Genetic variation and gastric cancer risk: A field synopsis and meta-analysis. Gut 64: 1209-1219, 2015.

7. Engreitz JM, Ollikainen $\mathrm{N}$ and Guttman M: Long non-coding RNAs: Spatial amplifiers that control nuclear structure and gene expression. Nat Rev Mol Cell Biol 17: 756-770, 2016.

8. Fatica A and Bozzoni I: Long non-coding RNAs: New players in cell differentiation and development. Nat Rev Genet 15: 7-21, 2014.

9. Shi X, Sun M, Liu H, Yao Y and Song Y: Long non-coding RNAs: A new frontier in the study of human diseases. Cancer Lett 339: 159-166, 2013.

10. Li J, Xuan Z and Liu C: Long non-coding RNAs and complex human diseases. Int J Mol Sci 14: 18790-18808, 2013.

11. Li X, Yu M, Chen L, Sun T, Wang H, Zhao L and Zhao Q: LncRNA PMS2L2 protects ATDC5 chondrocytes against lipopolysaccharide-induced inflammatory injury by sponging miR-203. Life Sci 217: 283-292, 2019.

12. Li BS, Zuo QF, Zhao YL, Xiao B, Zhuang Y, Mao XH, Wu C, Yang SM, Zeng H, Zou QM and Guo G: MicroRNA-25 promotes gastric cancer migration, invasion and proliferation by directly targeting transducer of ERBB2, 1 and correlates with poor survival. Oncogene 34: 2556-2665, 2015.
13. Yuan SQ, Chen YT and Huang ZP: Equipping the 8th edition American Joint Committee on Cancer Staging for Gastric Cancer with the 15-node minimum: A population-based study using recursive partitioning analysis. J Gastrointest Surg 21: 1-8, 2017.

14. Livak KJ and Schmittgen TD: Analysis of relative gene expression data using real-time quantitative PCR and the 2(-Delta Delta C(T)) method. Methods 25: 402-408, 2001.

15. Strong VE, Wu A, Selby LV, Gonen M, Hsu M, Song KY, Park CH, Coit DG, Ji JF and Brennan MF: Differences in gastric cancer survival between the US and China. J Surg Oncol 112: 31-37, 2015

16. Liu L, Yan B, Yang Z, Zhang X, Gu Q and Yue X: ncRuPAR inhibits gastric cancer progression by down-regulating protease-activated receptor-1. Tumour Biol 35: 7821-7829, 2014.

17. Deng T, Yuan Y, Zhang C, Zhang C, Yao W, Wang C, Liu R and $\mathrm{Ba}$ Y: Identification of circulating miR-25 as a potential biomarker for pancreatic cancer diagnosis. Cell Physiol Biochem 39: 1716-1722, 2016.

18. Feng X, Jiang J, Shi S, Xie H, Zhou L and Zheng S: Knockdown of miR-25 increases the sensitivity of liver cancer stem cells to TRAIL-induced apoptosis via PTEN/PI3K/Akt/Bad signaling pathway. Int J Oncol 49: 2600-2610, 2016.

19. Wu T, Chen W, Kong D, Li X, Lu H, Liu S, Wang J, Du L, Kong Q, Huang $X$ and Lu Z: miR-25 targets the modulator of apoptosis 1 gene in lung cancer. Carcinogenesis 36: 925-935, 2015.

20. Liang WC, Fu WM, Wong CW, Wang Y, Wang WM, Hu GX, Zhang L, Xiao LJ, Wan DC, Zhang JF and Waye MM: The lncRNA H19 promotes epithelial to mesenchymal transition by functioning as miRNA sponges in colorectal cancer. Oncotarget 6: 22513-22525, 2015.

21. Wu XS, Wang F, Li HF, Hu YP, Jiang L, Zhang F, Li ML, Wang XA, Jin YP, Zhang YJ and Lu W: LncRNA-PAGBC acts as a microRNA sponge and promotes gallbladder tumorigenesis. EMBO Rep 18: 1837-1853, 2017.

c) (i) (-) This work is licensed under a Creative Commons Attribution-NonCommercial-NoDerivatives 4.0 International (CC BY-NC-ND 4.0) License. 